\title{
INITIAL OPERATION OF THE MARYLAND COAXIAL GYROKLYSTRON EXPERIMENT
}

\author{
W. Lawson, M. Castle, J. Cheng, B. Hogan, G. P. Saraph, V. L. Granatstein, and M. Reiser, \\ Electrical Engineering Department and Institute for Plasma Research University of Maryland \\ College Park, MD 20742 USA.
}

\section{Abstract}

We describe results from the initial operation of our coaxial gyroklystron experiment, which is being evaluated as a potential driver for future linear colliders. The interaction is designed to occur between a $500 \mathrm{kV}$, $500-700$ A beam and a series of coaxial $\mathrm{TE}_{0 \mathrm{n} 1}$ microwave cavities. Output powers in excess of $100 \mathrm{MW}$ at $8.568 \mathrm{GHz}$ are expected with an efficiency of about $40 \%$ [1]. We detail performance of our single anode magnetron injection gun in addition to the stability and amplification properties of our preliminary microwave circuit. We also discuss our designs of near-term future tubes which are expected to have comparable performance at $17.136 \mathrm{GHz}$.

\section{INTRODUCTION}

At the University of Maryland, we have been running a comprehensive program to study the suitability of gyroklystrons as drivers for linear collider applications [1]. We have previously reported a variety of experimental results, all of which were achieved on a test bed which produced a small-orbit beam with a nominal voltage and current of $450 \mathrm{kV}$ and $200 \mathrm{~A}$, respectively. Published accounts of our effort include an amplified power level of $27 \mathrm{MW}$ at $32 \%$ efficiency in a three-cavity first harmonic gyroklystron near $10 \mathrm{GHz}$ [2] and $32 \mathrm{MW}$ at $29 \%$ efficiency in a two-cavity second harmonic gyroklystron near $20 \mathrm{GHz}$ [3].

In this paper we present the design details of two coaxial gyroklystron tubes which are predicted to produce at least $100 \mathrm{MW}$ of output power with an efficiency of nearly $40 \%$. These tubes utilize a fundamental mode $\mathrm{TE}_{011}$ input cavity which is driven by a $150 \mathrm{~kW}$ magnetron at $8.568 \mathrm{GHz}$. The first tube also has an $8.568 \mathrm{GHz} \mathrm{TE}_{011}$ output cavity, whereas the second tube has $17.136 \mathrm{GHz}$ $\mathrm{TE}_{021}$ buncher and output cavities. We present details of all system aspects, including the test bed modifications required to produce the enhanced beam characteristics, simulated beam properties, and simulated circuit interactions. Cold and hot test results of the first experimental tube are discussed before the project status and a description of our future plans are summarized.

\section{TEST BED MODIFICATIONS}

We have just completed an upgrade of our facility which should enable us to produce amplified microwave powers in excess of $100 \mathrm{MW}$ (see Fig. 1). Our modulator voltage and current have been increased to $500 \mathrm{kV}$ and $800 \mathrm{~A}$, respectively. We have designed, installed, and completed acceptance testing of a single-anode Magnetron Injection Gun (MIG) which is capable of producing a $480-720 \mathrm{~A}$ rotating electron beam at the nominal beam voltage with an axial velocity spread less than $7 \%$. The simulated space-charge-limited perveance of $5.5 \mu \mathrm{P}$ was in good agreement with the measured result. The maximum current produced in the acceptance test was limited by our modulator (due to an applied voltage which was lower than the nominal operating voltage) to $670 \mathrm{~A}$.

The original water-cooled magnets have been used, but a larger power supply for the gun coil was required because of a decrease in the magnetic compression. We reduced our drive frequency from $10 \mathrm{GHz}$ to exactly three times the current SLAC frequency, so a new coaxial magnetron and a modified input waveguide were required. The output waveguide (uptapers, beam dump,

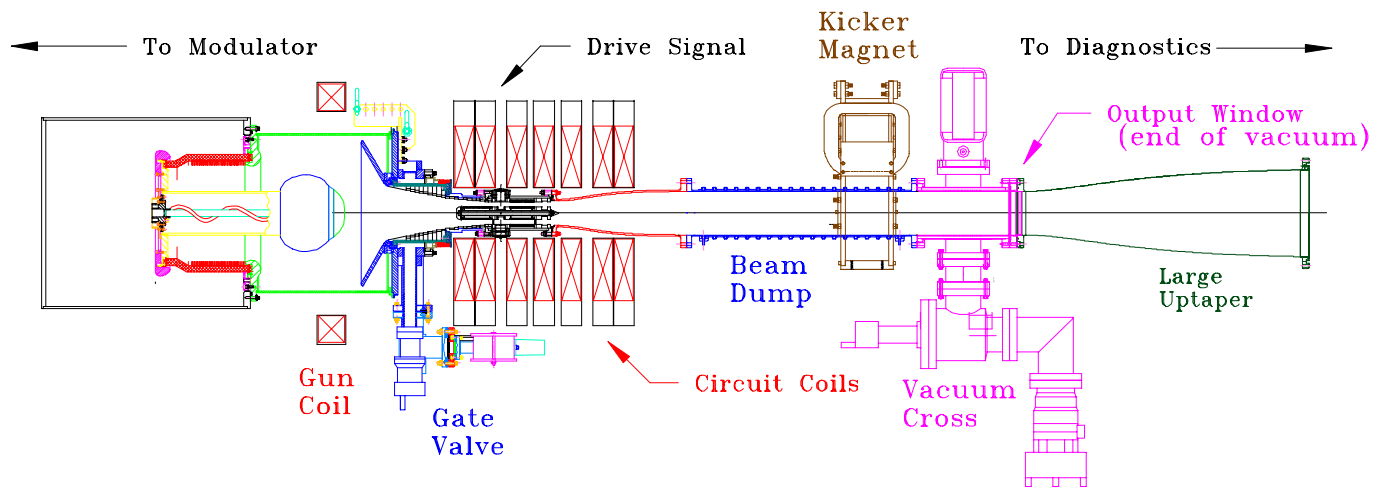

Fig. 1. The gyroklystron test bed. 
window, kicker magnet, pumping cross) was totally rebuilt to accommodate the expected larger peak powers. The anechoic chamber was modified to accommodate the new output waveguide and the directional coupler diagnostic was completely redesigned.

\section{THEORETICAL CIRCUIT PERFORMANCE}

A detailed design analysis has been carried out on a number of coaxial, two- and three-cavity gyroklystron systems with the aid of our partially self-consistent nonlinear code. The input cavity in all tubes is in resonance with the signal frequency at $8.568 \mathrm{GHz}$ and the output cavity is resonant with either the first $(8.568 \mathrm{GHz})$ or the second harmonic $(17.136 \mathrm{GHz})$ frequency. In a three-cavity system, an additional buncher cavity is introduced which is resonant at either the first or second harmonic frequency. In the following sections, we describe only the first harmonic tube which has been hottested and the second harmonic tube which is scheduled to undergo hot testing in the near future.

\subsection{Two-Cavity First Harmonic Tube}

The first harmonic tube consists of an input cavity and an output cavity separated by a drift section. The input cavity is defined by a decrease in the inner conductor radius only and the quality factor is brought down to $Q \approx$ 70 by loading the cavity with a thin ring of carbonized aluminum-silicate placed at one end of the cavity. The inner radius is $1.05 \mathrm{~cm}$ and the length is $2.29 \mathrm{~cm}$. Power is injected through two radial coupling ports which are separated by $180^{\circ}$ and excited in phase. Our startoscillation code predicts that the input cavity is completely stable up to a current of $800 \mathrm{~A}$.

The drift section has inner and outer radii of $1.825 \mathrm{~cm}$ and $3.325 \mathrm{~cm}$, respectively. The inner conductor is required so that the drift tube is cutoff to the operating mode. The regions adjacent to each cavity are made of copper, but lossy ceramics line the majority of the drift tube to eliminate spurious modes. The total length of the drift region is $9.1 \mathrm{~cm}$. Lossy ceramics are also used in the downtaper between the gun and the input cavity.

The output cavity is defined by changes in both radii and has a length of $1.70 \mathrm{~cm}$. Power is extracted axially into the output waveguide via a coupling aperture. The aperture has the same radii as the drift tube and has a length of $0.9 \mathrm{~cm}$. The diffractive quality factor is about 122. The start-oscillation code also predicts the output cavity to be stable at the nominal current, which is given in the middle column of Table 1 along with the other operating parameters. The efficiency is nearly $40 \%$ and the output power is about $95 \mathrm{MW}$. The dependence of tube efficiency on axial velocity spread is plotted in Fig. 2 with the solid line. The simulated velocity spread of the electron gun is $6.4 \%$ at the nominal current. The curve shows a slow but steady decrease in efficiency with increasing spread and indicates that an efficiency of $37 \%$ is still possible if the spread is as high as $10 \%$. A simplified schematic of the tube dimensions along with the optimal axial magnetic field profile is indicated in Fig. 3.

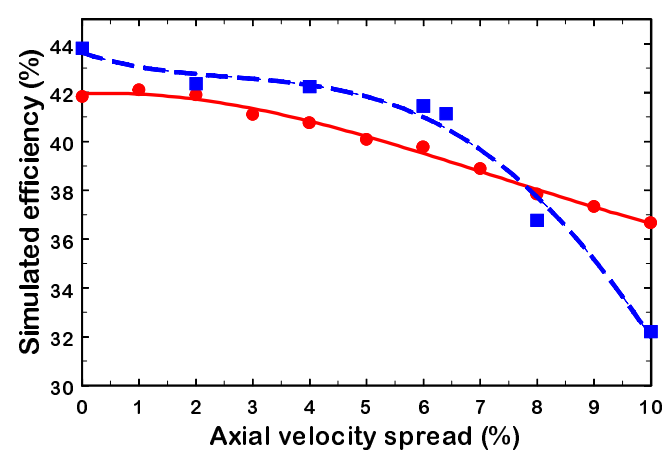

Fig. 2. Efficiency of the first (solid line) and second harmonic (dashed line) tubes vs. velocity spread.

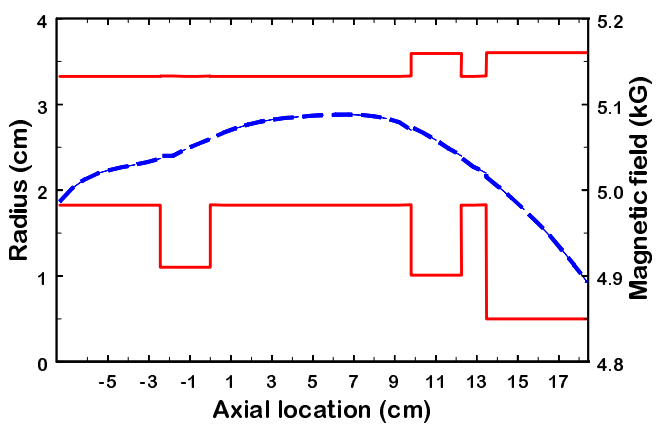

Fig. 3. The first-harmonic two-cavity tube and the optimal magnetic field profile.

\subsection{Three-Cavity Second Harmonic Tube}

The second harmonic design which we intend to test is a three-cavity system. The buncher cavity operates at the second harmonic and is formed with non-adiabatic radial wall transitions. Mode conversion from the $\mathrm{TE}_{02}$ mode to the $\mathrm{TE}_{01}$ is estimated to be about $-40 \mathrm{~dB}$. Dielectric loading of the cavity is used in order to obtain a Q of 389 and is achieved by reducing the thickness of the copper sections that separate the cavity from the drift tube dielectrics. The linear start oscillation code indicates that the buncher cavity is stable to beam currents below about $1000 \mathrm{~A}$ at the design value of the magnetic field $\left(\mathrm{B}_{0}=\right.$ $4.81 \mathrm{kG})$. The output cavity is also designed with nonadiabatic radial wall transitions. The scattering matrix code estimates the purity of the $\mathrm{TE}_{02}$ operation in the output cavity to be $97 \%$. The ratio of the power flowing 
into the drift tube to the power flowing into the output waveguide is better than $-24 \mathrm{~dB}$. Furthermore, the lossy dielectric loading in the drift tube, which will have a minimum effect on the Q-value of the operating mode, will suppress the excitation of the spurious modes and reduce the cross-talk.

TABLE 1. Comparison of the $1^{\text {st }}$ and $2^{\text {nd }}$ harmonic designs.

\begin{tabular}{||l|c|c||}
\hline \hline Parameters & $1^{\text {st }}$ harmonic & $2^{\text {nd }}$ harmonic \\
\hline Voltage & $500 \mathrm{kV}$ & $500 \mathrm{kV}$ \\
Current & $480 \mathrm{~A}$ & $770 \mathrm{~A}$ \\
Velocity ratio & 1.508 & 1.508 \\
Input Cavity Q & 50 & 50 \\
Buncher Cavity Q & - & 389 \\
Output Cavity Q & 122 & 320 \\
Gain & $21 \mathrm{~dB}$ & $49 \mathrm{~dB}$ \\
Efficiency & $39.4 \%$ & $41.1 \%$ \\
Output Power & $94.6 \mathrm{MW}$ & $158.2 \mathrm{MW}$ \\
\hline \hline
\end{tabular}

The simulated results at the nominal operating point are indicated in the final column of Table 1. The optimal current according to the simulations is $770 \mathrm{~A}$ and the estimated peak output power is over $150 \mathrm{MW}$. The corresponding gain and efficiency are $49 \mathrm{~dB}$ and $41 \%$, respectively. The dependence of efficiency on velocity spread is shown as the dashed line in Fig. 2. Note that the efficiency begins to drop off fairly rapidly for spreads above 7\%. However, these simulations are not reoptimized with respect to magnetic field profile, etc., at each point, and additional investigations indicate that higher efficiencies can be achieved if the velocity spread is higher than expected.

\section{COLD AND HOT TEST RESULTS}

The construction, cold testing, and hot testing of the first experimental tube has been completed. Cold-testing yielded the final dimensions of the input cavity required to achieve the frequency of $8.568 \mathrm{GHz}$ and a quality factor of 70. They are quite near the theoretical estimates given in the previous section. Cold-test drift tube attenuation measurements have indicated adequate isolation. The results for the output cavity were also quite close to the predicted values.

The performance of the microwave amplification experiment was limited by an input power coupling problem that developed after the tube was installed on the test bed. The net result was that we were only able to inject about $5 \mathrm{~kW}$ of power into the input cavity. The two cavity system had a predicted gain slightly above $20 \mathrm{~dB}$, so the tube was severly gain limited. The best amplification results yielded a peak power of about 600 $\mathrm{kW}$ with a pulse width of over $1.5 \mu \mathrm{s}$ at a beam voltage of $270 \mathrm{kV}$ and a current of $290 \mathrm{~A}$. The performance at this reduced operating point was consistant with our simulations. While some instabilities were observed in the output waveguide, performance of the tube at these beam parameters was not limited by spurious modes.

\section{PROJECT STATUS AND FUTURE PLANS}

We have nearly completed a rebuild of the first harmonic tube which incorporates two major changes. First we have added a third port on the vacuum jacket at the input cavity so that we can use transmission measurements to characterize the input cavity parameters in situ. Second have have lenghtened the overall system in order to add a buncher cavity so that the tube should not be gain limited. The buncher cavity is nearly identical in shape to the drive cavity and has the same resonant frequency and Q. We expect this tube to go on-line early in June 1997.

We continue to work on improving our simulation capabilities. Time-dependent capability has been added to our nonlinear (single-mode) code by researchers from the Naval Research Laboratory and initial results have confirmed the steady-state code predictions. We hope in the future to add multi-mode capability to our time dependent code.

We are also looking at advanced cavity concepts for future tubes. For the input cavity, we are using the High Frequency Structure Simulator code HFSS to simulate a single waveguide injection scheme which couples to the input cavity via an outer coaxial cavity. Furthermore, we are investigating an output cavity which couples through the inner radial wall to a circular waveguide, thereby decoupling the microwaves and the beam beyond the output cavity and enabling the use of additional tube stabilization schemes. Preliminary HFSS simulations of this output cavity scheme have been successful. Both concepts promise to improve performance of the gyroklystron tubes beyond the current predictions.

Finally, we are beginning to look at the application of gyroklystron circuits to Ka-band and beyond.

\section{ACKNOWLEDGEMENTS}

This work was supported by the Department of Energy.

\section{REFERENCES}

[1] Granatstein, V. L. and W. Lawson, "Gyro-Amplifiers as Candidate RF Drivers for TeV Linear Colliders," IEEE Trans. Plasma Sci. 24, 648 - 665 (1996).

[2] Tantawi, S., W. Main, P. E. Latham, G. Nusinovich, W. Lawson, C. D. Striffler, and V. L. Granatstein, "High Power X-Band Amplification from an Overmoded Three-Cavity Gyroklystron with a Tunable Penultimate Cavity," IEEE Trans. Plasma Sci. 20, 205 - 215 (1992).

[3] Matthews, H. W., W. Lawson, J. P. Calame, M. K. E. Flaherty, B. Hogan, J. Cheng, and P. E. Latham, "Experimental Studies of Stability and Amplification in a Two-Cavity Second Harmonic Gyroklystron," IEEE Trans. Plasma Sci. 22, 825 - 833 (1994). 